\title{
An attempt to evaluate the role of hearing in the social play of juvenile rats
}

\author{
JAMES A. HAGEMEYER and JAAK PANKSEPP \\ Bowling Green State University, Bowling Green, Ohio
}

\begin{abstract}
In one experiment, we attempted to evaluate the role of hearing in juvenile play by inducing deafness in young rats using a single subcutaneous injection of aminooxyacetic acid (AOAA) and kanamycin as described by Bryant et al. (1984). This treatment did not affect play, nor did a regimen of multiple injections. However, evaluation of auditory responsiveness indicated that these manipulations did not produce clear deafness. We then attempted to produce deafness using the AOAA/kanamycin injection in much younger rats and found the treatment to be lethal, a result that was due to the AOAA rather than the kanamycin. The AOAA dosage was then reduced to the minimal dose that Bryant had found to yield cochlear damage in guinea pigs, but our evaluation of acoustic startle indicated that auditory competence was not reduced in our rats. Clearly, this technique does not appear useful for producing deafness in rats, and the role of hearing in play remains to be adequately evaluated.
\end{abstract}

Social play, a prominent juvenile behavior of many mammalian species, has recently been studied from a psychobiological perspective (Panksepp, Siviy, \& Normansell, 1984; Thor \& Holloway, 1984). Social play consists of multiple behavior patterns during which a great variety of sensory stimulation is received and reacted to in rapid succession. The extent to which sensory input mediates play is not well understood, but the roles of a few systems have been experimentally analyzed. Neither vision (Bierley, Hughes, \& Beatty, 1986) nor olfaction (Beatty \& Costello, 1983; Thor \& Holloway, 1982) appears to be required for social play in rats. On the other hand, somatosensory information appears to be important for normal play. Play, as measured by pinning, is reduced markedly by anesthetization of the anterior dorsal body surface of rats (Siviy \& Panksepp, 1987). Interruption of audition was also reported to reduce play; however, this conclusion was based on pilot data, using animals in which hearing was physically precluded. Siviy and Panksepp (1987) plugged the ears of experimental subjects with wax and glued the pinnae shut; no comparable physical manipulation was done to the control animals. It is possible that the physical discomfort of this procedure on the experimental animals, rather than deafness, decreased play. Accordingly, in Experiment 1, our aim was to evaluate the role of hearing in play, using a physically less invasive procedure.

It has long been known that chronic exposure to antibiotics can cause deafness via destruction of cochlear hair cells. Kanamycin has been the most widely used ototoxic agent, but it is not clear how effective it is in rats. This manipulation has been found to produce deafness in rats,

Address correspondence to Jaak Panksepp, Department of Psychology, Bowling Green State University, Bowling Green, OH 43403. but 40 daily injections of $400 \mathrm{mg} / \mathrm{kg}$ kanamycin per rat were required (Harpur \& D'Arcy, 1976), which would take young rats past puberty and, hence, would not be especially useful for the study of juvenile play. Additionally, the repeated injection of ototoxic antibiotics causes such health related problems as weight loss and kidney malfunction. Because play behavior is typically reduced by illness, and because levels of play decline during puberty (Panksepp et al., 1984), the kanamycin technique for induction of deafness, which requires prolonged multiple injections, is far from ideal. Recently Bryant et al. (1984) reported that a single injection of $400 \mathrm{mg} / \mathrm{kg}$ kanamycin given concurrently with $8-25 \mathrm{mg} / \mathrm{kg}$ aminooxyacetic acid (AOAA) is sufficient to cause deafness in guinea pigs. Leake, Snyder, and Schreiner (1987) found this procedure also to be highly effective in cats. Deafness occurs within several hours and does not appear to create any of the health problems associated with multiple kanamycin injections. To our knowledge, this procedure has yet to be used in rats. If this procedure could successfully produce deafness in rats, it would be possible to evaluate the influence of deafness on juvenile play, without many of the confounds associated with animals deafened by physical means. In the first experiment, using young rats, we evaluated a dose and injection regimen of AOAA and kanamycin that had been proven effective in guinea pigs and cats.

\section{EXPERIMENT 1}

Bryant et al. (1984) found that a single dose of $8-25 \mathrm{mg} / \mathrm{kg}$ AOAA in combination with $400 \mathrm{mg} / \mathrm{kg}$ kanamycin was sufficient to induce deafness in guinea pigs. Our Experiment 1 was designed to determine whether play and hearing of juvenile rats are affected by this treatment. 


\section{Method}

Subjects. Three litters of male and female Long-Evans juvenile rats $(n=22)$ were used. The animals were bred and born at the Bowling Green State University animal facility and housed in a colony room maintained at $25^{\circ}-27^{\circ} \mathrm{C}$, with a 12:12-h light:dark cycle. The rats were group-housed with their litter mates and mothers in $24 \times 40 \times 19 \mathrm{~cm}$ suspended solid-bottom cages, with wood chip bedding. At weaning (Postnatal Day 24), animals were rehoused individually in $23 \times 10 \times 13 \mathrm{~cm}$ suspended wire-bottom cages. Throughout testing, food and water were available ad lib. Animals were paired according to sex and weight, and play pairs were maintained constant for all test days. Rats were weighed daily, and were initially injected on Postnatal Day 27 with $200 \mathrm{mg} / \mathrm{kg}$ kanamycin and $12.5 \mathrm{mg} / \mathrm{kg}$ AOAA (for the experimental group), or simply with $12.5 \mathrm{mg} / \mathrm{kg}$ AOAA (for the control group). In an attempt to maximize damage to cochlear hair cells, the rats were given five subsequent higher dose injections $(400 \mathrm{mg} / \mathrm{kg}$ kanamycin and $25 \mathrm{mg} / \mathrm{kg}$ AOAA or simply $25 \mathrm{mg} / \mathrm{kg}$ AOAA) on Postnatal Days $31,32,34,36$, and 37 (as indicated in Figure 1).

Apparatus. Play testing was conducted in an aluminum-walled $31 \times 31 \times 32 \mathrm{~cm}$ test box, with a clear acrylic front, situated on a Lafayette Instruments activity monitor (Model 201) that was tuned to selectively detect and automatically record the rough-and-tumble activity of the play pairs. The test box was situated in a sound-attenuated, ventilated chamber that was illuminated by a single $25-\mathrm{W}$ red light bulb mounted to one side. Specific play behaviors were observed through a $15 \times 15 \mathrm{~cm}$ observation window. The startle apparatus consisted of a small animal holder that was mounted onto a sensitive pressure transducer. A .22 caliber starter's pistol was used to generate startle stimuli.

Procedure. At 25 days of age ( 1 day following weaning and isolate housing), animals were given a 5 -min habituation session in the play chamber. When animals were 27 days of age, play testing was initiated. Animals were observed during the first half of the light cycle, in 5-min "paired-encounter" play sessions. Pins, dorsal contacts, and pin durations (as previously defined by Panksepp et al., 1984; Siviy \& Panksepp, 1987) were manually recorded by the observer, using a digital counter and running time meters. Following two baseline pretreatment tests at 25 and 26 days of age, animals were injected with the respective treatments described above. Two hours after these injections, animals were given a 5-min play test, and then tested at 24-h intervals for 3 days. Animals were reinjected with higher doses starting at 32 days of age (as indicated in Figure 1), and they were given daily play sessions that occurred $24 \mathrm{~h}$ following all subsequent injections.

Hearing was evaluated in three ways. (1) When the rats were 34 days of age, an audiotape of synthesized sirens was played for 1 min (at $86 \mathrm{~dB}$ SPL) during the 2 nd and 4 th $\min$ of the 5-min play session. (2) When the rats were 39 days of age, the acoustic startle response of each rat was evaluated. (3) Finally, at the end of the startle test, we observed the behavior of all rats in their home cages during the rattling of a chain of keys (a stimulus known to contain ultrasonic frequencies that agitate rats).

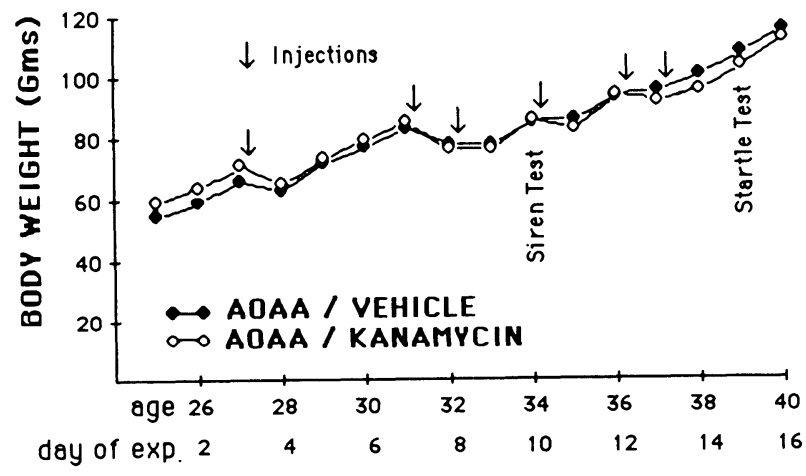

Figure 1. Daily mean weights of the AOAA/Vehicle- and AOAA/kanamycin-injected rats in Experiment 1. The arrows indicate when injections were given, after the daily play tests. Siren and startle test days are indicated.
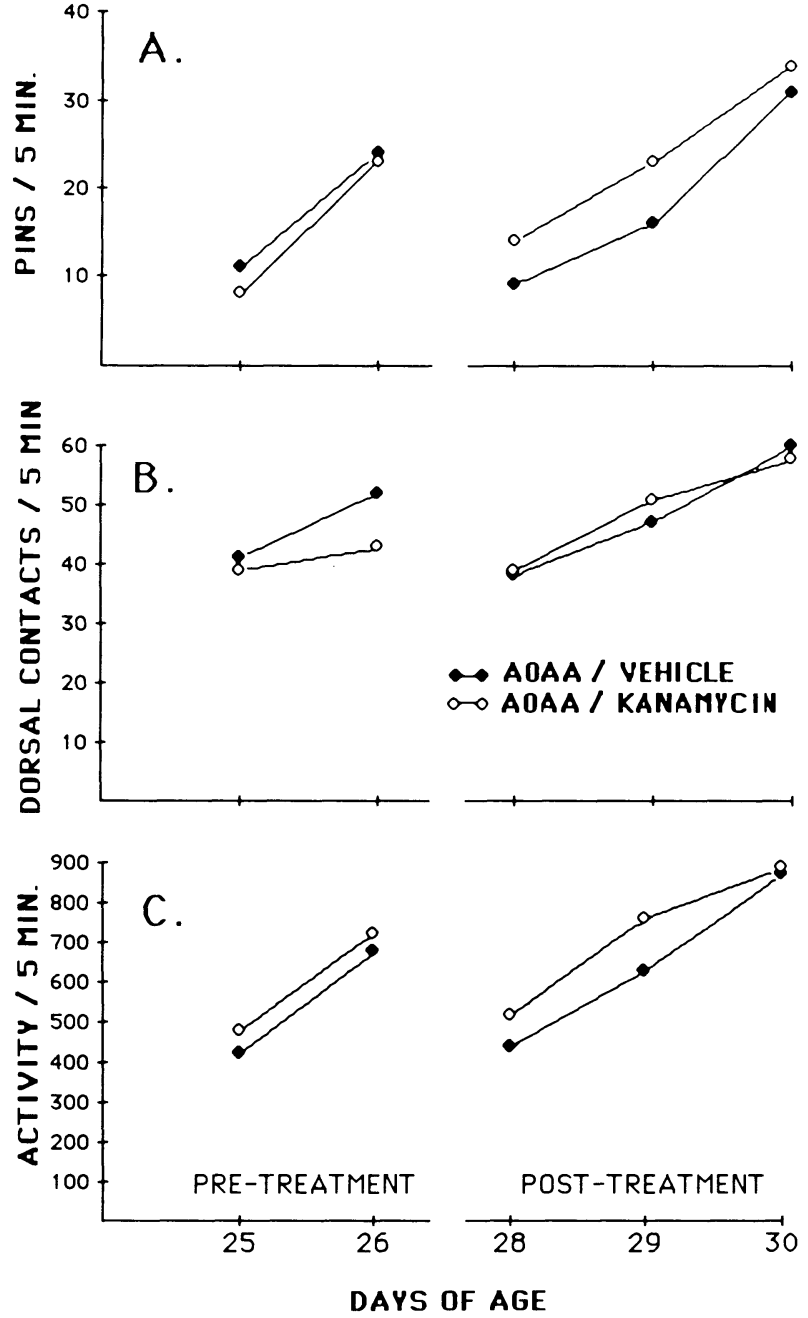

Figure 2. Mean number of pins (A), dorsal contacts (B), and overall rough-and-tumble activity $(C)$ during a 5-min play session are shown for the AOAA/vehicle- and AOAA/kanamycin-injected rats of Experiment 1. The first two data points represent the 2 days of baseline testing. The first drug injection was given on Day 27, followed by 3 consecutive days of testing. Additional tests following subsequent injections did not yield any differences between groups.

\section{Results}

The injections produced unambiguous sedation in all animals, controls and experimentals, which indicates that the effect was due to AOAA rather than to the kanamycin. This effect lasted about $6 \mathrm{~h}$. When animals were tested for play $2 \mathrm{~h}$ after the injections, none exhibited any play behaviors. Body weights for experimental and control animals (summarized in Figure 1) indicated that the two treatments had no differential effects on growth, but it should be noted that, following each injection, there was either a small reduction or no change in body weight. Since animals of this age typically grow a few grams each day, it can probably be concluded that, even in the absence of a non-AOAA control, AOAA inhibited eating during the subsequent day (an effect that has been observed with intracerebral administration of the drug; Panksepp \& Meeker, 1980). 
Play data for the 2 baseline days and the first 3 days of postdrug testing are summarized in Figure 2. Clearly, the animals that received AOAA and kanamycin played at least as much as did the animals that had been treated only with AOAA. The slight increase in play of the experimental group was not statistically reliable. The additional play test periods are not completely summarized since the same pattern was observed throughout all subsequent testing - namely, mean number of pins for the experimental group was never lower than for the control group. For the control and experimental groups, respectively, mean pins for these play sessions were 27 and 32 at 32 days of age, 24 and 26 at 33 days, 17 and 19 at 36 days, and 10 and 16 at 38 days.

The effect of noise (sirens) on play is summarized in Figure 3. As is evident, the noise reliably inhibited play $[F(1,14)=16.6, p<.001]$, but there was no reliable difference between groups and no group $x$ noise interaction. The ability of noise to inhibit play was reliable in both groups $(p<.05)$. Startle tests affirmed that the kanamycin-treated animals were not deaf. All animals exhibited clear, and essentially identical, acoustic startle responses. In response to the firing of the starter's pistol, the average transient force exerted by control animals was $65 \mathrm{~g}$, and by experimental animals was $64 \mathrm{~g}$. Also, all animals clearly responded to manual jiggling of keys (i.e., the ultrasonic test) in front of their home cage.

\section{Discussion}

Although hearing loss could be induced by coadministration of AOAA and kanamycin to guinea pigs and cats, we found no such effect in juvenile rats. Considering how resistant rats appear to have been to the ototoxic effects of kanamycin in past work (Harpur \& D'Arcy, 1976), we would provisionally conclude that the combined treatment with AOAA and kanamycin is not an especially effective manipulation for producing deafness in rats. This conclusion is further highlighted by the fact that the treatment could be administered repeatedly without apparent effect. One noteworthy observation is that AOAA, by itself, seemed to severely debilitate our young animals for several hours. During this period, no playful behavior was observed. The only positive finding of this experiment was that noise (i.e., simulated sirens) could reliably

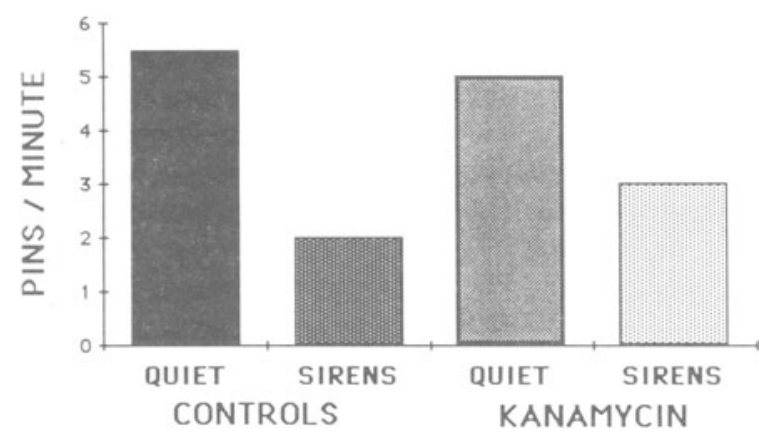

Figure 3. Average pin counts/min for the multiple AOAA/vehicleand AOAA/kanamycin-injected rat pups used in Experiment 2 during ambient noise (sirens at $86 \mathrm{db}$ ) and during silence are summarized. During a 5-min play session, 1 min of baseline data was collected alternating with 1 min of sirens. The average scores for the respective conditions are provided. reduce play behavior, and this is probably because of the rats' temporary shift in attention due to the distraction.

\section{EXPERIMENT 2}

To further determine whether the AOAA/kanamycin technique could be employed in rats, we evaluated the effects of such treatments in much younger animals. In preliminary testing, a single injection of $25 \mathrm{mg} / \mathrm{kg}$ of AOAA was found to be lethal to 12- to 16-day-old rat pups. Since young rats could survive $8 \mathrm{mg} / \mathrm{kg}$ of AOAA, which was a highly effective dose in guinea pigs (Bryant et al., 1983), we decided to combine this dose with $400 \mathrm{mg} / \mathrm{kg}$ of kanamycin. Unfortunately, of 16 animals that were given this treatment for 3 successive days between 12 and 14 days of age, only 6 animals survived. The animals seemed to be deaf when tested for startle, and their growth had been severely retarded, indicating other severe effects on development than mere ototoxicity. Accordingly, in the following experiment, we utilized the parameters of treatment that would optimize survival and subsequent growth of young rat pups, namely, two injections of $8 \mathrm{mg} / \mathrm{kg}$ of AOAA in combination with $400 \mathrm{mg} / \mathrm{kg}$ of kanamycin.

\section{Method}

Subjects and Apparatus. Two litters of male and female Long-Evans derived rat pups were randomly assigned to two groups and given consecutive s.c. injections of $400 \mathrm{mg} / \mathrm{kg}$ kanamycin and $8 \mathrm{~kg} / \mathrm{mg}$ AOAA or simply $8 \mathrm{mg} / \mathrm{kg}$ AOAA when they were 12 and 13 days of age. The apparatus used was the same as in Experiment 1.

Procedure. At 23 days of age, the pups were weaned and individually housed, and play pairs were made. On Postnatal Day 27, play testing began, using the same procedures as in Experiment 1. At 29 days of age, subjects were evaluated for deafness using the startle and ultrasonic tests described in Experiment 1.

\section{Results}

The play data are summarized in Figure 4. Pins were found to be slightly, albeit significantly, lower in experimental animals than in control animals $[F(1,16)=$ $10.2, p<.01$, but no reliable differences were apparent in dorsal contacts $[F(1,16)=.62]$ or the overall measure of rough and tumble activity $[F(1,16)=.05]$. Each measure exhibited an age effect: Pins increased as animals aged $[F(6,96)=7.1, p<.001]$, dorsal contacts exhibited a slight increase followed by a slight reduction $[F(6,96)$ $=3.7, p<.01]$, and activity also showed a systematic increase $[F(5,35)=12.6, p<.001]$.

Acoustic startle data yielded no differences in auditory responsiveness between the two groups. All animals exhibited a clear startle response, as measured by the pressure detector. The animals also responded to the jingling of keys.

\section{Discussion}

As with the previous experiment, we were unable to evaluate the role of hearing in play because the AOAA/kanamycin treatment did not totally deafen the neonatal rats, and partial hearing loss cannot be evalu- 

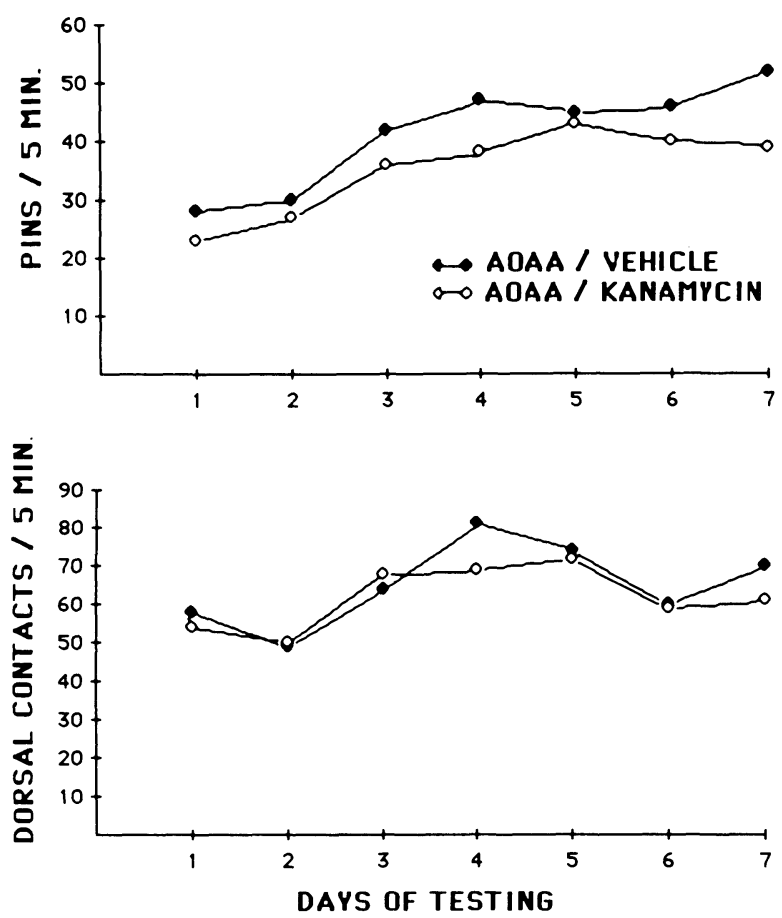

Figure 4. Pin numbers (top figure) and dorsal contacts (bottom figure) for AOAA/vehicle- or AOAA/kanamycin-injected rat pups used in Experiment 2 during 5-min play sessions across 7 days. Only pinning was marginally reduced in the AOAA/kanamycin animals.

ated from the present data. The fact that we observed hearing impairments in a pilot group of rats that were given an LD $\mathbf{5 0}$ of these drugs suggests that the margin of safety between ototoxicity and survival in young rats is small indeed. Accordingly, we would conclude that this strategy is not optimal for evaluating the role of hearing in the play of juvenile rats. At the present time, we cannot specify the reason for the slight reduction in pinning in the experimental group. The pattern, though modest, is similar to that which Siviy and Panksepp (1987) reported for physically deafened animals (i.e., a reduction in pins with no change in the dorsal contact measure of play solicitation.) Since rough-and-tumble activity was not reduced at all, the reduction in pins probably does not reflect a change in play motivation, but only a slight change in the consummatory phase of play.

\section{GENERAL DISCUSSION}

Although the conjoint administration of AOAA and kanamycin in a single dose has been found to be a useful maneuver for producing deafness in guinea pigs and cats (Bryant et al., 1984; Leake et al., 1987), this treatment does not appear to be as effective in rats. Although deafness can probably be produced by near lethal doses of AOAA and kanamycin, this does not appear to be an optimal approach for addressing the question of auditory functions in play. Accordingly, it is likely that the role of hearing in play will have to be evaluated by some other procedure, probably direct surgical intervention in auditory processing.
A subsidiary, and unanticipated, finding of the present work was that AOAA rather than kanamycin is the toxic ingredient in the above cocktail. Overall, the AOAA appeared to be considerably more toxic in young neonatal rats (those tested in Experiment 2) than in the older juveniles (those in Experiment 1). The older (post-30-day-old) rats were able to tolerate at least three times the dose that killed younger (12- to 16-dayold) animals. Still, the older animals did exhibit a global toxic response to AOAA. For several hours following treatment, they were lethargic and exhibited no motivation to play. To our knowledge, this behavioral effect of AOAA has not been reported in previous work that used this agent as a promoter of the ototoxic effects of kanamycin, and it should be taken into consideration whenever short-term studies are planned. For instance, in the Leake et al. (1987) paper, auditory-evoked potentials were essentialy eliminated $2 \mathrm{~h}$ after treatment, but no description of the health status of the animals was provided.

A subsidiary finding of the present experiment is that noise can temporarily reduce play. Although this finding proved to be an adequate way to evaluate the auditory capacity of rats, it is not an especially important effect. It is not surprising that an attention-diverting stimulus would temporarily disrupt an ongoing behavior pattern. In additional tests, we have evaluated the effects of loud music presented throughout a 5-min play period; except for a slight transitory inhibition of play at the outset of the session, no overall reduction of playful activity could be detected. Accordingly, we would conclude that sound, as long as it remains nonthreatening, has little effect on the playful tendencies of young rats.

\section{REFERENCES}

Bryant, G., Cronin-Schreiber, R., Alexander, A., Norris, C., QUINE, D., \& GUTH, P. (1984). The potentiation of ototoxicity when aminooxyacetic acid and kanamycin are co-administered. Hearing Research, 15, 173-178.

BeatTy, W. W., \& Costello, K. B. (1983). Olfactory bulbectomy and play fighting in juvenile rats. Physiology \& Behavior, 30, 525-528.

Bierley, R. A., Hughes, S. L., \& Beatty, W. W. (1986). Blindness and play fighting in juvenile rats. Physiology \& Behavior, 36, 199-201 .

HARPUR, E. S., \& D'ARCY, P. (1976). Comparison of the ototoxic effect of kanamycin on albino and pigmented rats, studied using an operant method. Experientia, 1562-1564.

Leake, P. A., SNyder, R. L., \& Schreiner, C. E. (1987). Cochlear pathology of sensorineural deafness in cats: Coadministration of kanamycin and aminooxyacetic acid. Annals of Otolaryngology, Rhinology and Laryngology, 1987, 96(Suppl. 128), 48-50.

PANKSEPP, J., \& MEeKer, R. B. (1980). The role of GABA in the ventromedial hypothalamic regulation of food intake. GABA Neurotransmission, Brain Research Bulletin, 5(Suppl. 2), 453-460.

Panksepp, J., SiviY, S., \& Normansell, L. (1984). The psychobiology of play: Theoretical and methodological perspectives. Neuroscience \& Behavioral Reviews, 8, 465-492.

SiviY, S., \& PANKSEPP, J. (1987). Sensory modulation of juvenile play in rats. Developmental Psychobiology, 20, 39-55.

ThOR, D. H. , \&olloway, W. R. (1982). Anosmia and play fighting behavior in prepubescent male and female rats. Physiology \& Behavior, 29, 281-285.

ThOR, D. H., \& HollowaY, W. R. (1984). Social play in juvenile rats: A decade of methodological and experimental research. Neuroscience \& Biobehavioral Reviews, 8, 455-464.

(Manuscript received for publication February 3, 1988.) 